\title{
Evaluation of an Amniotic Membrane-Collagen Dermal Substitute in the Management of Full-Thickness Skin Defects in a Pig
}

\author{
Hyunji Kim ${ }^{1}$, Daegu Son 2 , Tae Hyun $\mathrm{Choi}^{3}$, Samhyun Jung ${ }^{4}$, Sunyoung Kwon 5 , Junhyung Kim², \\ Kihwan $\operatorname{Han}^{2}$ \\ ${ }^{1}$ Suibi Plastic Surgery Clinic, Daegu; ${ }^{2}$ Department of Plastic and Reconstructive Surgery, Keimyung University Dongsan Medical Center, \\ Keimyung University School of Medicine, Daegu; ${ }^{3}$ Department of Plastic and Reconstructive Surgery, Seoul National University College of \\ Medicine, Seoul; ${ }^{4}$ Department of Tissue Engineering, Bioland, Cheongwon; ${ }^{5}$ Department of Pathology, Keimyung University Dongsan \\ Medical Center, Keimyung University School of Medicine, Daegu, Korea
}

Background To minimize the inflammatory reaction and improve healing, a new modified dermal substitute composed of an atelocollagen, chondroitin-6-sulfate, and amniotic membrane (AM) was applied to full-thickness skin defects in a pig. Atelocollagen was extracted from bovine skin, and two modified dermal substitutes were generated according to the crosslinking type.

Methods The AM-collagen dermal substitutes were characterized and compared with currently used dermal substitutes in a pig skin defect model. There were five experimental groups: dehydrothermal (DHT) cross-linking atelocollagen with the AM on the top (AM-DHT), DHT and chemical cross-linking atelocollagen with the AM on the top (AM-DHT/chemical), Terudermis, Integra, and AlloDerm. After $3 \times 3 \mathrm{~cm}$ full-thickness skin defects on the back of a pig were created, each dermal substitutes dermal substitutes was randomly grafted on the defects. Two weeks after grafting, autologous partial-thickness skin was over-grafted on the neodermis. The take rate of the dermal substitutes, skin, and histological sections were all assessed at 1, 2, and 4 weeks postoperatively.

Results More rapid healing and a higher take rate were evident in the AM-DHT and Terudermis groups. Histological examination revealed fewer inflammatory cells and more fibroblast hyperplasia in these two groups. Four weeks after surgery, the amount of newly formed collagen was significantly more appropriate in the AM-DHT group.

Conclusions These observations provide supporting evidence that a newly developed amnioticcollagen dermal substitute may inhibit inflammatory reactions and promote wound healing.

Keywords Amnion / Dermis / Biologic dressings / Collagen / Chondroitin sulfates
Correspondence: Daegu Son Department of Plastic and Reconstructive Surgery, Keimyung University Dongsan Medical Center, Keimyung University School of Medicine, 56 Dalseong-ro, Jung-gu, Daegu 700-712, Korea

Tel: +82-53-250-7636

Fax: +82-53-255-0632

E-mail: handson@dsmc.or.kr

This article contains supplemental Figs. S1-S4.

This article has been adapted from Hyunji Kim's dissertation submitted to Keimyung University Graduate School for the Ph.D. in Plastic Surgery.

This article was presented at the 57 th Congress of the Korean Society of Plastic and Reconstructive Surgeons on November 13, 2004 in Seoul, Korea.

No potential conflict of interest relevant to this article was reported.

\section{INTRODUCTION}

Up to the present, autologous skin grafts or flaps have been wide- ly used for repairing skin and soft tissue defects. However, improved reconstruction poses greater donor site problems. To resolve these problems, skin substitutes have been developed using

Copyright () 2013 The Korean Society of Plastic and Reconstructive Surgeons

This is an Open Access article distributed under the terms of the Creative Commons Attribution Non-Commercial License (http://creativecommons.org/

licenses/by-nc/3.0/) which permits unrestricted non-commercial use, distribution, and reproduction in any medium, provided the original work is properly cited.

www.e-aps.org 
tissue engineering [1]. However, in order for skin substitutes to be applied to patients with full-thickness skin loss, the substitutes should function as an alternative to autologous skin, form an effective barrier against bacterial invasion, minimize inflammation and scar formation, improve fibrovascular tissue ingrowth, and have excellent reproducibility $[2,3]$.

A dermal substitute, which is the most extensively developed of the current skin substitutes, consists of a collagen scaffold that comprises mainly collagen containing either dermis extracellular matrix or cultured fibroblasts. A bilayered skin substitute that contains epidermis-forming keratinocytes attached on a collagen scaffold with cultured fibroblasts is ideal. However, its take rate is problematic.

We have generated a novel dermal substitute containing a collagen scaffold by combining chondroitin-6-sulphate (C6S) with atelocollagen (ATC) extracted from calf dermis. To lessen the antigenicity of ATC, the telopeptides located at the terminal of the collagen molecule were removed using pepsin. C6S was added to increase stability and the scaffold was treated by physical cross-linking in a dehydrothermal (DHT) treatment alone or with an additional chemical cross-linking involving 1-ethyl3-(3-dimethylaminopropyl) carbodiimide (EDC). We used an amniotic membrane (AM) instead of silicone membranes that temporarily served as epidermis, to protect the wound from the external environment, to keep the wound moist, and to aid in the promotion of wound healing through various growth factors.

This study was undertaken to generate a new dermal substitute and to compare it with previously designed dermal substitutes, in order to confirm its usefulness in clinical practice through animal experiments.

\section{METHODS}

\section{Preparation of an atelocollagen dermal substitute Purification of ATC solution}

The extracted collagen from calf skin was treated with $2 \mathrm{~mol} / \mathrm{L}$ $\mathrm{NaCl}$ and agitated, and the pellets were obtained using centrifugation (6,000 rpm for 20 minutes). The pellets were dissolved in $1 \mathrm{~mol} / \mathrm{L} \mathrm{NaCl} / 0.05 \mathrm{~mol} / \mathrm{L}$ Tris at $\mathrm{pH} 7.4$, and the supernatant was obtained using centrifugation (3,500 rpm for 60 minutes). The supernatant was salted out for 3 hours with an addition of 4 $\mathrm{mol} / \mathrm{L} \mathrm{NaCl}$, and the pellets were retrieved using centrifugation $(6,000 \mathrm{rpm}$ for 20 minutes). The pellets were dissolved in a $\mathrm{pH}$ $2.0 \mathrm{HCI}$ solution, treated with $2 \mathrm{~mol} / \mathrm{L} \mathrm{NaCl}$, and agitated. After centrifugation $(6,000 \mathrm{rpm}$ for 20 minutes) the pellets were again dissolved in the HCI solution. The solution was dialyzed in a dialysis tube and retrieved. After titration at $\mathrm{pH} 3$, the solution was filtered using a $0.2 \mu \mathrm{m}$ filter to obtain $0.5 \%$ ATC.

\section{Preparation and cross-linking of AM-collagen scaffold}

The AM was harvested from a healthy bovine placenta and disinfected with $70 \%$ ethanol and $0.05 \%$ sodium hypochlorite. During the processing, the epidermis was removed and the remaining stromal layer was incubated in an aqueous solution containing $0.25 \%(\mathrm{w} / \mathrm{v})$ trypsin, $0.02 \%(\mathrm{w} / \mathrm{v})$ ethylene diamine tetraacetic acid, and $0.9 \%(\mathrm{w} / \mathrm{v}) \mathrm{NaCl}$ at $\mathrm{pH} 7.4$ for 60 minutes to remove the cellular components. After the cells were removed from the tissue, the acellular AMs were dried to preserve their biochemical and structural integrity. The purified 0.5\% ATC was mixed with $1 \%$ C6S equivalent to $10 \%$ of the dried weight of the collagen and was agitated at $1,500 \mathrm{rpm}$ at $4^{\circ} \mathrm{C}$ for $5 \mathrm{~min}$ utes. The cream-colored ATC-C6S solution was poured into a square-shaped disk mold, in which an AM $(12.5 \times 12.5 \mathrm{~cm})$ was attached using a cell scraper. The bubbles were completely removed using an air drier, and the collagen scaffold was frozen at $-80^{\circ} \mathrm{C}$ and then lyophilized for 48 hours.

The lyophilized collagen scaffold was cut into $3 \times 3 \mathrm{~cm}$ sections using a surgical scalpel. Each section was placed in a bottle, and DHT cross-linking was performed in a vacuum oven at $110^{\circ} \mathrm{C}$. For additional chemical cross-linking, the sections were treated with an EDC solution at $\mathrm{pH} 5.5$, rinsed sufficiently with a 0.1 $\mathrm{moL} / \mathrm{L} \mathrm{NaHPO}_{4}$ solution, and hydrated with purified water. The sections were then frozen at $-80^{\circ} \mathrm{C}$ and dried using a lyophilizer. The ATC dermal substitute was sealed using an air-permeable material and sterilized with ethylene oxide gas.

\section{Characterization of the AM-collagen dermal substitute} Amino acid analysis

The ATC solution $(1.31 \mathrm{mg})$ was hydrolyzed and labeled with phenylisothiocyanate (PITC) using a Pico-Tag Kit (Waters Associates, Milford, MA, USA). The chromatograms were obtained from $10 \mu \mathrm{L}$ aliquots of the $1,600 \mu \mathrm{L}$ volume of the PITClabeled specimens by high performance liquid chromatography (Waters Associates).

\section{Electron microscopy}

Both DHT cross-linked and DHT/chemical cross-linked collagen scaffolds were treated with gold using a Polaron E5100 Coating System (JEOL, Tokyo, Japan) and their cut surface was examined by scanning electron microscopy (SEM) using a JSM6300 microscope (JEOL) at a magnification of $\times 150$.

\section{Biochemical analysis of amniotic membrane}

Freeze dried bovine placental amniotic membrane was used as raw material before making the AM-collagen dermal substitutes and processed $\mathrm{AM}$ was used for biochemical analysis. Two kinds of tissue samples were frozen in liquid nitrogen, ground with a 
freezer mill (SamplePrep Model 6750, SPEX, Metuchen, NJ, USA), and homogenized in $1 \mathrm{~mL}$ of phosphate-buffered saline. The protein levels were measured using an enzyme linked immunosorbent assay system (fibroblast growth factor [FGF], DFB50; keratinocyte growth factor [KGF], DKG00; R\&D Systems, Minneapolis, MN, USA). The total proteins were measured with the Bradford assay.

\section{Animal experiments}

The experiments were conducted in 12 2- to 3-month-old Yorkshire female pigs free of skin diseases. The animals were sedated using an intramuscular injection of $2.2 \mathrm{mg} / \mathrm{kg}$ azaperone (Stresnil, Korea Yansen, Seoul, Korea), and the skin was prepared. Xylazine $\mathrm{HCl}$ (3 mg/kg; Rumpun, Bayer Korea, Seoul, Korea) and ketamine $\mathrm{HCl}$ ( $8 \mathrm{mg} / \mathrm{kg}$; Ketalar, Yuhan Yanghang Pharmaceuti$\mathrm{cal}$, Seoul, Korea) were administered through the auricular vein.

\section{Creation of full-thickness skin defects and application of the dermal substitutes}

The site of the wound was designated with a marking pen on the skin over the paravertebral muscle of the pig, with a square size of $30 \mathrm{~mm}$. The full-thickness skin above the fascia of the muscle was excised with a No. 10 scalpel (Fig. 1). Two kinds of the novel AM-collagen dermal substitute (group 1 and 2) and three other groups of currently available dermal substitutes were prepared: group 1, DHT cross-linking atelocollagen scaffold with the AM on one side (AM-DHT group); group 2, DHT and chemical cross-linked atelocollagen scaffold with AM on one side (AM-DHT/chemical group); group 3, the Terudermis group (Terumo, Tokyo, Japan); group 4, the Interga group (LifeSciences, Plainsboro, NJ, USA); and group 5, the AlloDerm group (LifeCell, Woodlands, TX, USA) (Table 1). Five different types of dermal substitutes from the five experimental groups were all randomly grafted into the full-thickness of every pig on

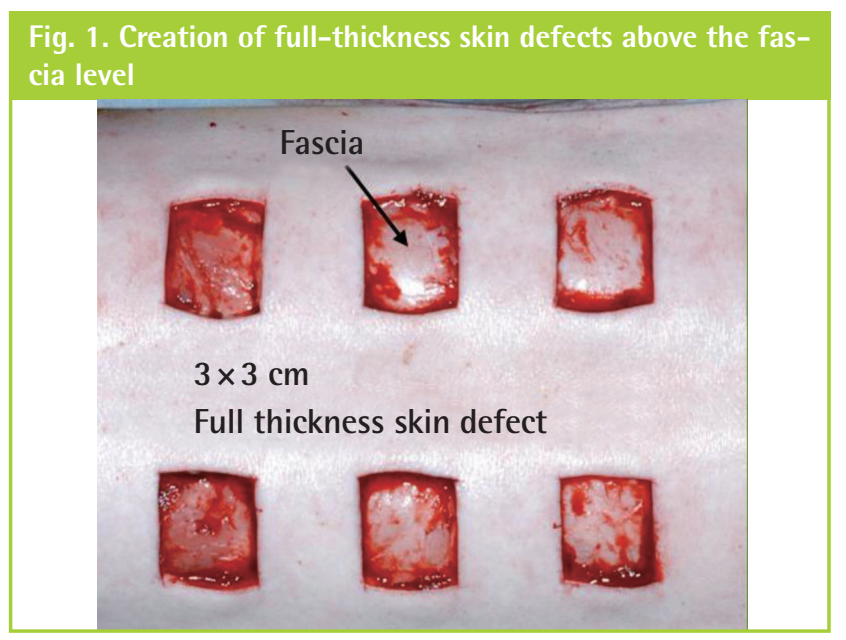

its skin defects. The surgical wound was draped with Allevyn and Opsite (Smith \& Nephew, London, UK). The dressings were further fixed with cotton balls and a plastic cover and were changed every 3 days. Twelve pigs were operated on sequentially under the same protocol and were categorized into three equal groups of four according to time of examination $(n=4)$. Each group was euthanized for biopsy on postoperative weeks 1, 2, and 4 after inspection and gross examination. For the animals in the 4-week group, autologous partial-thickness skin from the groin area was applied to the neodermis 2 weeks after the dermal substitute grafting in order to ascertain whether the neodermis was vascularized enough to accept a free skin graft.

\section{Gross findings and histological examination}

The take rates of the dermal substitute grafts of the five groups were assessed at 1 and 2 weeks after grafting. With the 4-week pig group, the partial-thickness skin grafts that had been applied to the neodermis, which were induced by the dermal substitutes at 2 weeks, were assessed for the take rate. Excluding the principal investigator and co-investigators of this study, five residents of the Department of Plastic and Reconstructive Surgery of our hospital who were blind to this study rated the take rate of the grafts using an ordinary scale method. An 8-point scale was used, with 1 being worst and 8 being best. Each of the scores were totaled and categorized as excellent (score of 31 to 40), good (score of 21 to 30), fair (score of 11 to 20), or poor (score of $\leq 10)$.

After the animals were euthanized, the grafted dermal substitutes were harvested for histological examination including the underlying muscle and fascia and $10 \mathrm{~mm}$ of normal skin around the wound edge. Four specimens $(n=4)$ were obtained from

Table 1. Summary of the experimental groups according to composition

\begin{tabular}{|c|c|c|}
\hline Group & $\begin{array}{l}\text { Temporary epider- } \\
\text { mal component }\end{array}$ & $\begin{array}{l}\text { Dermal } \\
\text { component }\end{array}$ \\
\hline \multirow[t]{2}{*}{ Group 1 (AM-DHT) } & Amniotic membrane & $\begin{array}{l}\text { Atelocollagen and C6S } \\
\text { (bovine skin) }\end{array}$ \\
\hline & & DHT cross-linked \\
\hline \multirow[t]{2}{*}{$\begin{array}{l}\text { Group } 2 \\
\text { (AM-DHT/chemical) }\end{array}$} & Amniotic membrane & $\begin{array}{l}\text { Atelocollagen and C6S } \\
\text { (bovine skin) }\end{array}$ \\
\hline & & DHT/chemical cross-linked \\
\hline \multirow[t]{2}{*}{ Group 3 (Terudermis) } & $\begin{array}{l}\text { Synthetic polysiloxane } \\
\text { polymer }\end{array}$ & $\begin{array}{l}\text { Denatured atelocollagen and } \\
\text { fibrillar atelocollagen } \\
\text { (bovine skin) }\end{array}$ \\
\hline & & DHT cross-linked \\
\hline Group 4 (Integra) & $\begin{array}{l}\text { Synthetic polysiloxane } \\
\text { polymer }\end{array}$ & $\begin{array}{l}\text { Atelocollagen and C6S } \\
\text { (bovine tendon) Chemical } \\
\text { cross-linked }\end{array}$ \\
\hline Group 5 (AlloDerm) & None & $\begin{array}{l}\text { Acellular human dermal } \\
\text { tissue }\end{array}$ \\
\hline
\end{tabular}


each experimental group. The specimens were fixed in $10 \%$ neutral buffered formaldehyde and stained with hematoxylineosin, Masson's trichrome, and $\alpha$-SMA immunohistochemical staining. The specimens obtained 1 and 2 weeks after surgery were assessed for inflammatory cell infiltration, neovascularization, fibroblast hyperplasia, and the replacement extent of the dermal substitutes with the neodermis. The specimens obtained 4 weeks after surgery were assessed for the arrangement of collagen in the neodermis, and the degree of similarity between the grafted substitute and the normal skin was determined.

\section{RESULTS}

\section{Characterization of the AM-collagen dermal substitute Antigenicity of the ATC solution}

The antigenicity of the purified ATC was evaluated through the analysis of its amino acid composition (Table 2). The percentage of tyrosine relative to the total amino acids was $0.12 \%$, which was lower than that of a criterion for nonantigenic collagen $(0.2 \%)$ [4].

\section{Electron microscopy findings of the collagen scaffold}

The cut surfaces of representative collagen scaffolds were examined using SEM, and the structure of collagen fibers was examined using TEM. The DHT cross-linked atelocollagen scaffolds

Table 2. Analysis of amino acid composition in the atelocollagen

\begin{tabular}{|lc|}
\hline Amino acid & Composition (\%) \\
\hline Glycine & 33 \\
Proline & 12 \\
OH-proline & 10 \\
Tyrosinea) & 0.12 \\
Cysteine & $<0.1$ \\
\hline a) Tyrosine (\%), antigenicity of atelocollagen. & \\
\hline
\end{tabular}

Table 3. Clinical analysis of the take rates of the dermal substitute grafts at postoperative week 1 and 2, and the partialthickness skin grafts at postoperative week 4 (ordinary scale method, $n=4$ )

\begin{tabular}{|lccc|}
\hline \multirow{2}{*}{ Group } & \multicolumn{3}{c|}{ Postoperative (wk) } \\
\cline { 2 - 4 } & $\mathbf{1}^{\text {a) }}$ & $\mathbf{2}^{\text {a) }}$ & $\mathbf{4}^{\text {a) }}$ \\
\hline AM-DHT & 38 & 36 & 35 \\
AM-DHT/chemical & 28 & 31 & 32 \\
Terudermis & 37 & 35 & 34 \\
Integra & 24 & 28 & 33 \\
AlloDerm & 8 & 17 & 12 \\
\hline AM, amniotic membrane; DHT, dehydrothermal. & \\
a) Excellent, 40-31; good, 30-21; fair, 20-11; poor, $\leq 10$. & \\
\hline
\end{tabular}

revealed the cut surface of the collagen scaffolds to be similar to that of normal skin, but the DHT/chemical cross-linked atelocollagen scaffold exhibited a somewhat crushed appearance (Fig. 2).

\section{Biochemical analysis of the amniotic membrane}

Quantities of FGF in a freeze dried bovine placental AM and processed AM were $919.8 \mathrm{pg} / \mathrm{mg}$ and $41.7 \mathrm{pg} / \mathrm{mg}$, respectively. The amount of KGF was similar before and after cell exclusion, but the proportional levels of the extracted protein pre- and postcell removal were $31.01 \mathrm{pg} / \mathrm{mg}$ and $238.64 \mathrm{pg} / \mathrm{mg}$, respectively. In other words, the protein level was 8 times higher after cell extraction. Therefore, it was confirmed that KGF was preserved in the processed AM.

\section{Animal experiments \\ Gross findings}

One week after surgery, groups 1 and 3 showed better take rates of the dermal grafts than the other groups. Overall, the take rate was better in the DHT cross-linked group than in the DHT/ chemical cross-linked group (Fig. 3). Two weeks after surgery, the take rate of the dermal grafts was slightly better in the DHT cross-linked groups than in the DHT/chemical cross-linked groups, but the difference in the take rate had decreased considerably.

Four weeks after surgery, the take rates of the partial-thickness skin grafted on the neodermis induced by the dermal substitute were excellent in all of the groups with the exception of group 5 (Table 3).

\section{Histological findings}

One week after surgery, group 1 had scanty exudate under the AM, good fibroblast hyperplasia, and good neovascularization, and most of the undersurface of the collagen scaffold of the dermal substitute was replaced with newly formed dermis. Group 3

\section{Fig. 2. Electron microscopy findings of the collagen scaffold}

Representative scanning electron micrographs of the DHT crosslinked and DHT/chemical cross-linked collagen scaffolds $(\times 150)$. The DHT/chemical cross-linked collagen scaffold collapsed. (A) Cross section of group 1 (AM-DHT). (B) Cross section of group 2 (AM-DHT/ chemical). DHT, dehydrothermal; $A M$, amniotic membrane.
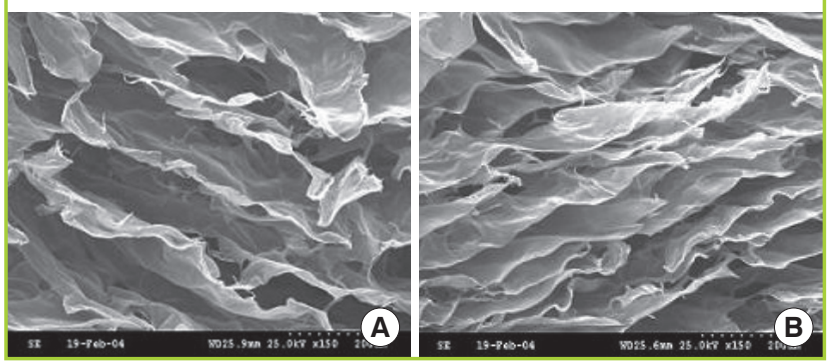


\section{Fig. 3. Macroscopic findings at postoperative week 1}

(A) AM-DHT, (B) AM-DHT/chemical, (C) Terudermis, (D) Integra, (E) AlloDerm. Healthier granulation tissue was seen through the graft site, and the grafts were less friable in group 1 and group $3(A, C)$. The take rates were higher in groups 1 and $2(A, B)$. AM, amniotic membrane; $\mathrm{DHT}$, dehydrothermal.
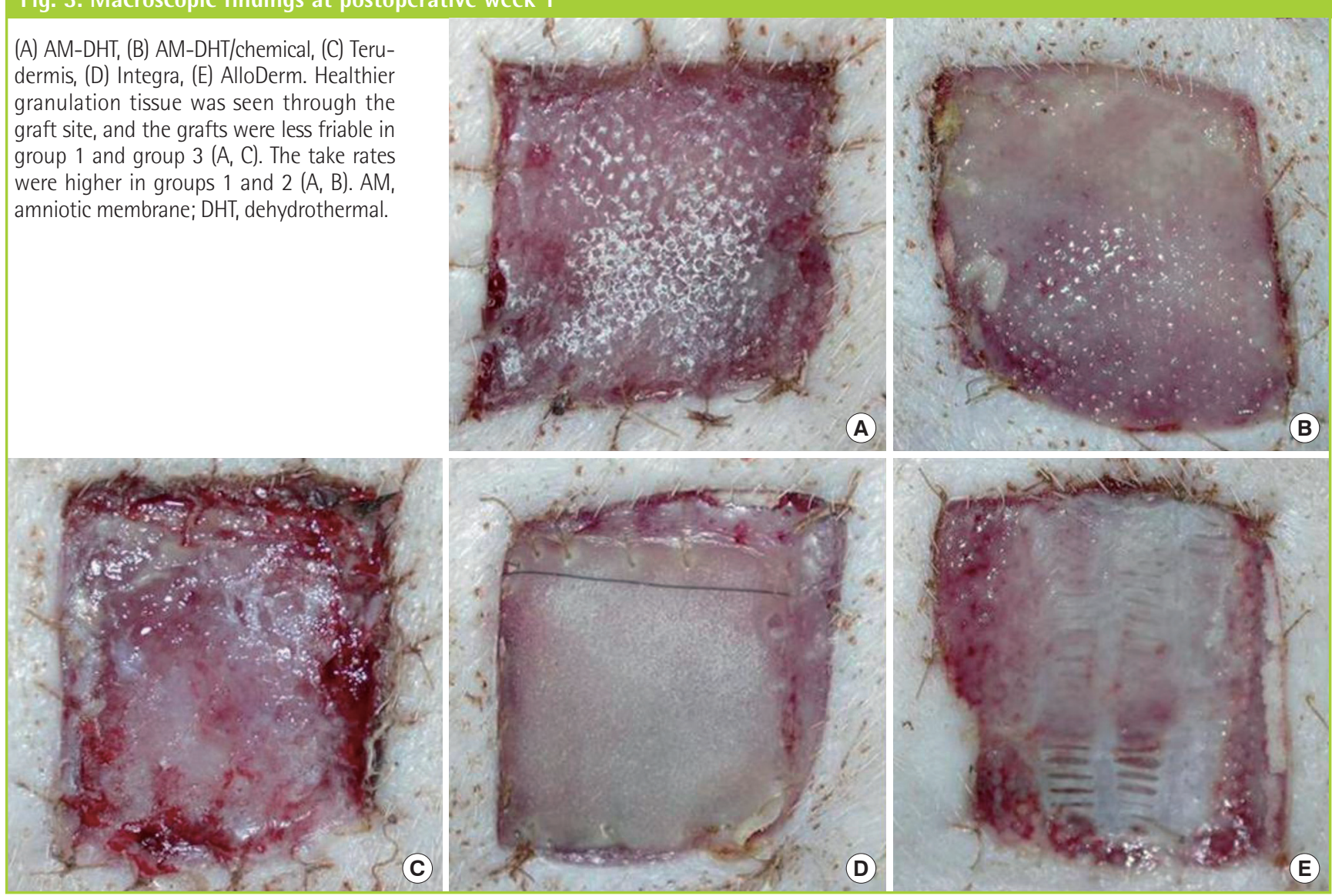

Table 4. Summary of the microscopic findings at postoperative weeks 1 and $2(n=4)$

\begin{tabular}{|c|c|c|c|c|c|}
\hline Group & AM-DHT & AM-DHT/chemical & Terudermis & Integra & AlloDerm \\
\hline \multicolumn{6}{|c|}{ Inflammatory cell infiltration (wk) } \\
\hline 1 & ++ & + & ++ & + & ++ \\
\hline 2 & + & + & + & + & ++++ \\
\hline \multicolumn{6}{|c|}{ Fibroblast proliferation (wk) } \\
\hline 1 & +++ & ++ & +++ & + & + \\
\hline 2 & ++++ & +++ & ++++ & ++ & + \\
\hline \multicolumn{6}{|c|}{ Angiogenesis (wk) } \\
\hline 1 & +++ & ++ & +++ & + & + \\
\hline 2 & ++++ & +++ & ++++ & ++ & + \\
\hline
\end{tabular}

also showed good fibroblast hyperplasia and good neovascularization on most of the collagen scaffold, except on a part of the upper surface. However, in group 2, a considerable amount of the grafted dermal substitute remained unchanged and showed fibroblast hyperplasia only on the undersurface of the collagen scaffold. In group 4, it remained unchanged and showed little inflammatory cell infiltration or delayed graft take at the undersurface of the collagen scaffold due to focal penetration of fibroblasts. Group 5 showed severe inflammatory cell infiltration and calcification (Fig. 4, Supplemental Fig. 1).
Two weeks after surgery, groups 1 and 3 showed remarkable fibroblast hyperplasia, good neovascularization, and good maturation of newly formed collagen. Groups 2 and 4 revealed good penetration of fibroblasts into the collagen scaffold and good fibroblast hyperplasia, whereas neovascularization was slightly delayed and the upper surface of the collagen scaffold remained partially unchanged. Group 5 showed poor inflammatory cell infiltration, severe calcification, and lymphadenoid changes (Table 4, Supplemental Fig. 2).

Four weeks after surgery, in groups 1 and 3 , the arrangement 
Fig. 4. Microscopic findings at postoperative week $1\left(H \& E_{1} \times 40\right)$

(A) AM-DHT, (B) AM-DHT/chemical, (C) Terudermis, (D) Integra, (E) AlloDerm. Fibrovascular tissue ingrowth was seen in group 1 and group $3(A, C)$. The collagen scaffold was slightly replaced with neodermis in group 3 and group 4 (C, D). AM, amniotic membrane; DHT, dehydrothermal.
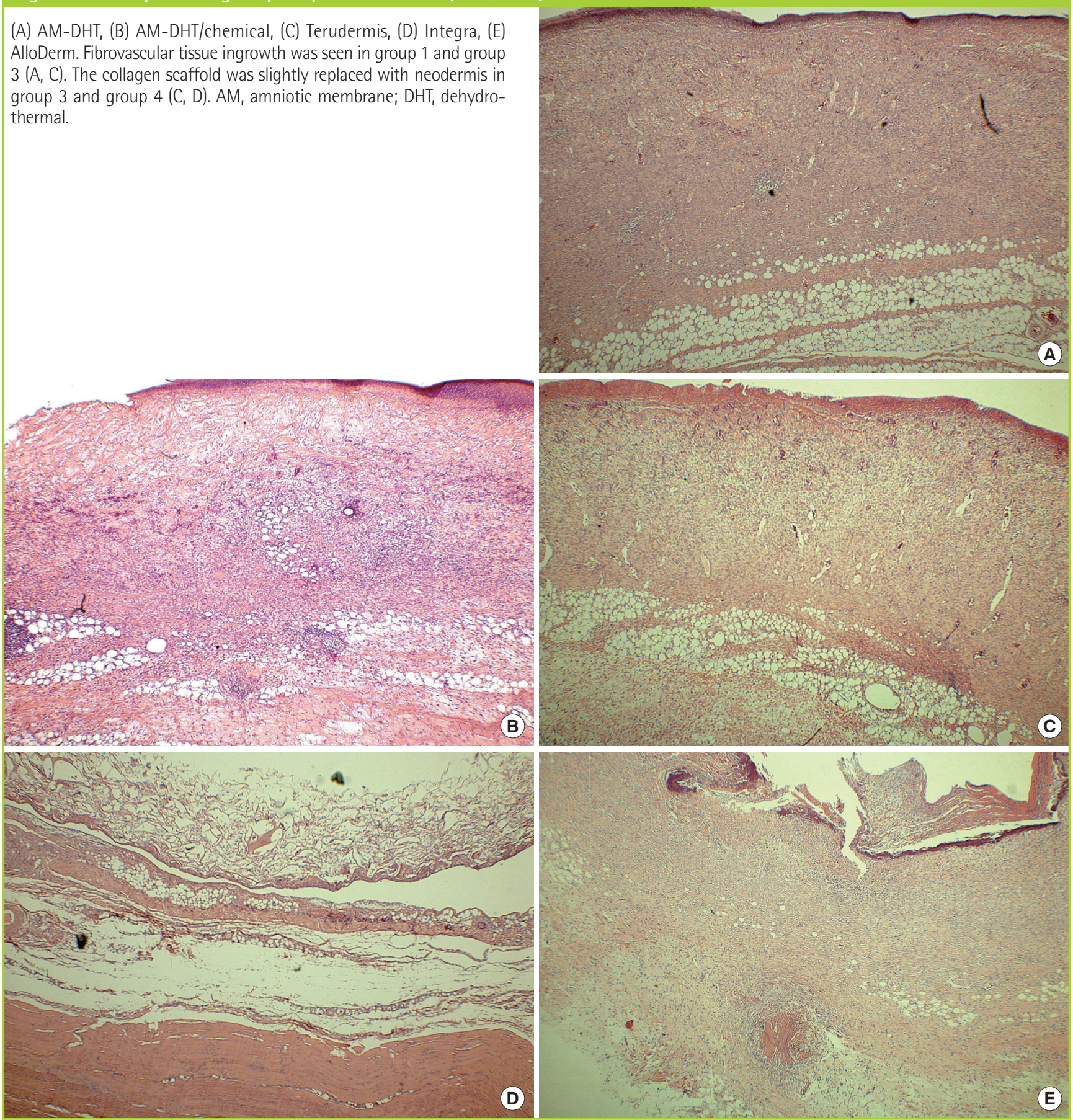

and amount of newly formed collagen were appropriate and similar to normal dermis, showing that the number of cells was small compared with the amount of collagen. The remaining groups showed similar findings but revealed a rather unstable arrangement of newly formed collagen fibers (Supplemental Figs. 3, 4).

\section{DISCUSSION}

Dermal substitutes should be physically strong and easy to han-

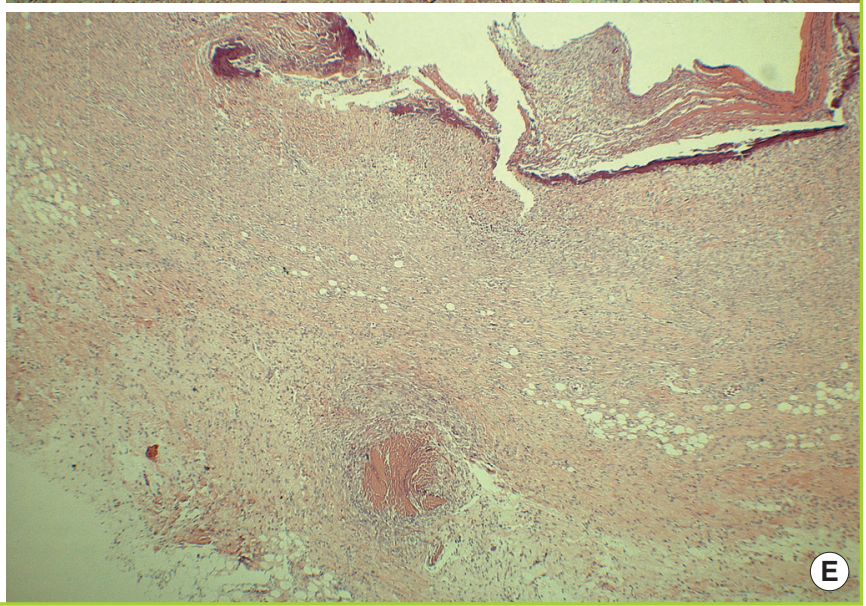

dle, endure contraction through durability, continue to attach viable fibroblasts during the whole wound healing process, enable the reconstruction of the dermal lattice with fibroblasts, and should endure hydrolysis during their presence in the wound until they are finally absorbed after complete wound healing $[2,5]$.

The use of a collagen scaffold as a dermal substitute reduces wound contraction and scar formation, and promotes epithelialization. Collagen has attracted much attention because it 
stimulates wound healing through its strong affinity to the soft tissue and blood vessels, and it can be replaced with host cells and vessels through acceleration of their penetration into the scaffold [6]. Because chemical and physical changes in collagen may affect the recipient tissue morphologically and physiologically, we attempted to investigate whether changes in collagen would affect its affinity to host cells.

In this study, the novel substitute displayed many advantages over substitutes currently in use. We used the AM instead of a silicone membrane to obtain the physical protection barrier functions as well as the anatomical and physiological functions similar to epidermis. The AM consists of three layers: the epithelial layer, basement membrane, and mesenchymal layer. The epithelial layer consists of a single layer of epithelial cells, and the basement membrane is a thin membrane composed of reticular fibers that attach to the epithelial layer by interdigitation. The mesenchymal layer is an avascular collagen matrix that is divided into compact, fibroblast, and sponge layers $[7,8]$. Although the AM has some variations in structure, it has histologically similar structures to the skin composed of the multilayered epithelium and basement membrane and, thus, has advantages in wound healing such as promotion of epithelialization, reduction in inflammatory reactions, and inhibition of scar formation and bacterial growth $[9,10]$. Promotion of epithelialization using AM has been clinically utilized for various skin lesions such as burns and ulcerations, and many growth factors have been identified in the AM by experimental studies. Koizumi et al. [11] documented that the AM expresses mRNAs of 8 growth factors that remarkably influence epithelial regeneration. Hao et al. [12] demonstrated that the AM shows mRNA expression of the interleukin-1 receptor antagonist and interleukin-10. In addition, Tseng et al. [13] suggested that inflammatory reactions are significantly reduced in the cornea, and limbus grown on the AM, probably due to reduced mRNA expression of transforming growth factor (TGF) receptor II and TGF- $\beta-1,2$, and 3 through the inhibition of the TGF- $\beta$-signaling system. Inhibition of the TGF- $\beta$ signaling system can reduce scar formation through the activation of fibroblasts into myofibroblasts. It has been reported that the amnion and chorion have an antibacterial effect against hemolytic Streptococcus group A, Escherichia coli, and Pseudomonas [14].

AM has been used as a temporary substitute because it protects skin wounds from the external environment, it decreases the loss of water, electrolytes, protein, and energy, it reduces pain, it compensates for the disadvantage of dermal substitutes in current use that are prone to infection, and it promotes wound healing through various growth factors [15].

We generated collagen scaffolds by combining C6S with atelo- collagen extracted from calf dermis. To lessen the antigenicity of the atelocollagen, the telopeptides located at the terminal of the collagen molecule were removed using pepsin. In this atelocollagen, the percentage of tyrosine in relationship to the total amino acids was $0.12 \%$, which was significantly lower than that of the criterion for non-antigenicity of collagen $(0.2 \%)$, suggesting that inflammatory reactions can be adequately lessened when grafted in human skin. C6S is a high-molecular substance that inhibits proteolytic enzymes, plays a crucial role in the binding and regulation of growth factors, and influences chemotaxis, migration, hyperplasia, and differentiation of cells [16]. Thus, the dermal substitute we generated by the addition of $\mathrm{C} 6 \mathrm{~S}$ could reduce biodegradation of collagen caused by proteolytic enzymes, and could lead to easy migration of cells from the surrounding tissue into this collagen scaffold due to the increased elasticity, durability, and porosity compared to a collagen scaffold alone [17].

DHT/chemical cross-linking inhibits the rapid degradation of a collagen scaffold, lessens its antigenicity, and enforces its physical properties when it is grafted on human skin. A DHT cross-linked collagen scaffold is more stable, maintains the same porosity as that prior to the treatment without distortion, and does not induce cytotoxic substances. This collagen scaffold is distorted in an EDC solution due to hydrophobicity induced by the initial DHT-treatment. Although this phenomenon can be prevented to some extent when crosslinking is conducted in the presence of ethanol, such chemical substances can induce cytotoxic substances in vivo. However, an EDC-treated collagen scaffold can prevent rapid degradation because it becomes resistant to proteolytic enzymes [16].

In this study, the results were significantly different between the experimental groups. Groups 1 and 2 (the DHT-treated groups) showed a more rapid wound healing than the other groups, suggesting that DHT-treated collagen scaffolds have a strong affinity to cellular components of the surrounding soft tissue and blood vessels, which was evident from the electron microscopic findings (Supplemental Fig. 3). The results support the idea that it is conceivable that atelocollagen may inhibit inflammatory reactions by lessening antigenicity, whereas $\mathrm{C} 6 \mathrm{~S}$ may promote wound healing by reducing biodegradation caused by proteolytic enzymes, which increases physical stability and induces the ready migration of cells from the surrounding tissue into the collagen scaffold through its increased porosity. In addition, AM used as a temporary protection membrane can promote wound healing, probably through the secretion of various growth factors.

In conclusion, our atelocollagen dermal substitute that was generated using atelocollagen, $\mathrm{AM}$, and C6S may be more reliable than collagen dermal substitutes in current use. 


\section{REFERENCES}

1. Boyce ST. Design principles for composition and performance of cultured skin substitutes. Burns 2001;27:523-33.

2. Balasubramani M, Kumar TR, Babu M. Skin substitutes: a review. Burns 2001;27:534-44.

3. Bannasch H, Fohn M, Unterberg T, et al. Skin tissue engineering. Clin Plast Surg 2003;30:573-9.

4. Yang EK, Park JK, Ahn JI, et al. Tissue-engineered skin substitutes using collagen scaffold with amniotic membrane component. Key Eng Mater 2005;277-279:12-8.

5. Koide M, Osaki K, Konishi J, et al. A new type of biomaterial for artificial skin: dehydrothermally cross-linked composites of fibrillar and denatured collagens. J Biomed Mater Res 1993;27:79-87.

6. Ono I, Tateshita T, Inoue M. Effects of a collagen matrix containing basic fibroblast growth factor on wound contraction. J Biomed Mater Res 1999;48:621-30.

7. Bourne GL. The microscopic anatomy of the human amnion and chorion. Am J Obstet Gynecol 1960;79:1070-3.

8. Young B, Heath JW. Wheater's functional histology: a text and colour atlas. 4th ed. Edinburgh: Churchill Livingstone; 2000.

9. Gruss JS, Jirsch DW. Human amniotic membrane: a versatile wound dressing. Can Med Assoc J 1978;118:1237-46.
10. Dua HS, Gomes JA, King AJ, et al. The amniotic membrane in ophthalmology. Surv Ophthalmol 2004;49:51-77.

11. Koizumi NJ, Inatomi TJ, Sotozono CJ, et al. Growth factor mRNA and protein in preserved human amniotic membrane. Curr Eye Res 2000;20:173-7.

12. Hao Y, Ma DH, Hwang DG, et al. Identification of antiangiogenic and antiinflammatory proteins in human amniotic membrane. Cornea 2000;19:348-52.

13. Tseng SC, Li DQ Ma X. Suppression of transforming growth factor-beta isoforms, TGF-beta receptor type II, and myofibroblast differentiation in cultured human corneal and limbal fibroblasts by amniotic membrane matrix. J Cell Physiol 1999; 179:325-35.

14. Kjaergaard N, Hein M, Hyttel L, et al. Antibacterial properties of human amnion and chorion in vitro. Eur J Obstet Gynecol Reprod Biol 2001;94:224-9.

15. Park M, Kim S, Kim IS, et al. Healing of a porcine burn wound dressed with human and bovine amniotic membranes. Wound Repair Regen 2008;16:520-8.

16. Pieper JS, Oosterhof A, Dijkstra PJ, et al. Preparation and characterization of porous crosslinked collagenous matrices containing bioavailable chondroitin sulphate. Biomaterials 1999;20:847-58.

17. Yannas IV, Burke JF. Design of an artificial skin. I. Basic design principles. J Biomed Mater Res 1980;14:65-81.

\section{Supplemental data can be found at: http://e-aps.org/src/sm/aps-40-11-s001.pdf}

